\title{
NLR singletons, pairs and networks: evolution, assembly and regulation of the intracellular immunoreceptor circuitry of plants
}

\author{
Hiroaki Adachi, Lida Derevnina, Sophien Kamoun† \\ The Sainsbury Laboratory, University of East Anglia, Norwich Research Park, Norwich, NR4 \\ $7 \mathrm{UH}, \mathrm{UK}$
}

† Correspondence to: sophien.kamoun@tsl.ac.uk

\begin{abstract}
NLRs are modular plant and animal proteins that are intracellular sensors of pathogenassociated molecules. Upon pathogen perception, NLRs trigger a potent broad-spectrum immune reaction known as the hypersensitive response. An emerging paradigm is that plant NLR immune receptors form networks with varying degrees of complexity. NLRs may have evolved from multifunctional singleton receptors, which combine pathogen detection (sensor activity) and immune signalling (helper or executor activity) into a single protein, to functionally specialized interconnected receptor pairs and networks. In this article, we highlight some of the recent advances in plant NLR biology by discussing models of NLR evolution, NLR complex formation, and how NLR (mis)regulation modulates immunity and autoimmunity. Multidisciplinary approaches are required to dissect the evolution, assembly and regulation of the immune receptor circuitry of plants. With the new conceptual framework provided by the elucidation of the structure and activationmechanism of a plant NLR resistosome, this field is entering an exciting era of research.
\end{abstract}

\section{Introduction}

Plants have a multi-layered immune system activated by receptor proteins that detect pathogen molecules and trigger an immune response. Nucleotide-binding domain and leucine-rich repeat (NLR) proteins make up one of the largest and widespread family of immune receptors. NLRs function inside plant cells by monitoring the activity of pathogen secreted molecules known as "effectors", which have evolved to facilitate pathogen spread and infection by modulating host processes. The recognition of pathogen effectors by host NLRs leads to NLR-triggered immunity (also known as effector-triggered immunity), a robust immune response that is often accompanied by localised cell death in the host known as the hypersensitive response (HR) $[1,2]$. In addition to plants, NLR-mediated immunity is an important component of the immune system of animals, and possibly fungi [3]. Unlike plants, animal NLRs tend to respond to pathogen molecular patterns rather than the rapidly evolving effectors $[3,4]$. Whether plant and animal NLRs share a common evolutionary origin has been under debate. Urbach et al. argued that the occurrence of NLRs across diverse eukaryotes is a result of convergent evolution [5]. However, the recent elucidation by cryo-electron microscopy (cryo-EM) of the structure of a plant NLR oligomer revealed a wheel-like pentameric resistosome with remarkable similarity to metazoan inflammasomes and 
apoptosomes [6], indicating that these divergent NLRs probably evolved from a common ancestor.

NLRs show a multi-domain structure and have a conserved nucleotide-binding (NB) domain and a C-terminal leucine-rich repeat (LRR) domain. In addition to the NB and LRR domains, most NLRs have characteristic N-terminal domains that are likely involved in immune activation [7]. Based on N-terminal domain features, plant NLRs can be broadly categorized into three subgroups, toll and interleukin-1 receptor (TIR)-type NLR (TIR-NLR), coiled-coil (CC)type NLR (CC-NLR) and RPW8-like CC-type NLR (RPW8-NLR) [8]. Recognition of effectors by plant NLRs follows multiple mechanistic models; "Direct", "Guard", "Decoy" and "Integrateddecoy" (reviewed by references 9 and 10). The "Integrated-decoy" model was revealed from functional analyse of unusual domains in several NLRs. These NLRs are referred to as "NLRID" based on their additional accessory domains called "integrated domains (IDs)" [11]. The IDs have a pivotal role in effector recognition and are proposed to have evolved by integration of effector host targets into the classical NLR domain architecture.

Recent progress in understanding plant immunity revealed that immune receptors, including NLRs, can form networks of varying complexity that translate pathogen detection into activation of hypersensitive cell death and immunity $[12,13]$. However, despite notable advances in NLR biology, including the recent elucidation of the structure and activation mechanism of the resistosome [6,14], how NLRs and their networks are regulated at multiple levels remains largely unknown. In this review, we highlight some of the recent advances in plant NLR biology by discussing models of NLR evolution and functional specialization, NLR complex formation, and how NLR regulation modulates immunity and autoimmunity.

\section{NLR evolution: from singleton to pairs to network}

The conceptual basis of host-pathogen interactions was first described by Harold Henry Flor in his gene-for-gene model. Flor's model postulated that inheritance of resistance and parasitism is determined by matching single genes in plants and pathogens [15]. Fifty years afterFlor proposed his gene forgene model, the first $R$-gene in plants were cloned [10]. To date dozens of $R$ genes have been cloned and remarkably the majority of these $R$ genes encode NLR proteins. True to Flor's model, some NLRs indeed function as a single genetic unit for sensing and signalling, referred to here as "singleton NLR" (Figure 1a, Table 1). These NLRs sense effectors either directly or indirectly and trigger the so-called hypersensitive immune response in the host. MILDEW LOCUS A (MLA) protein family, found broadly across different barley accessions, is a well-studied NLR locus required for isolate-specific resistance to powdery mildew fungi [16]. MLA directly recognizes matching avirulence effector, AVRa, and triggers cell death in the heterologous Nicotiana benthamiana system, suggesting that it probably behaves both as a sensor and as a signal inducer [17]. Other likely singleton NLRs that could sense effectors and independently trigger hypersensitive cell death in heterologous plant systems include Sr50, a rye ortholog of MLA, L6, a flaxNLR, and some Arabidopsis NLRs such as RESISTANCE TO PERONOSPORA PARASITICA 13 (RPP13), RESISTANCE TO PSEUDOMONAS SYRINGAE5 (RPS5) and HOPZ-ACTIVATED RESISTANCE1 (ZAR1) [18-24]. 
Recent findings revealed that the functional principles of NLR-mediated immunity are more complex than previously anticipated with many NLRs requiring other NLR proteins to function $[25,26]$ (Figure 1a, Table 2). Some NLRs work in pairs, in which a sensor NLR, specialized to recognize the pathogen, is coupled with a helper (also known as executor) NLR that is involved in initiating immune signalling [27]. In Arabidopsis and rice, genetically linked NLR pairs, RESISTANCE TO RALSTONIA SOLANACEARUM 1 (RRS1)/RESISTANCE TO PSEUDOMONAS SYRINGAE 4 (RPS4), RESISTANCE GENE ANALOG 5 (RGA5)/RGA4 and PYRICULARIA ORYZAE RESISTANCE K-1 (Pik-1)/Pik-2 function together. In these pairs, RRS1, RGA5 and Pik-1 are typical NLR-IDs, they possess additional domains for effector recognition, WRKY domain in RRS1 and HEAVY METAL-ASSOCIATED (HMA) domain in both RGA5 and Pik-1 and require RPS4, RGA4 and Pik-2, respectively, to trigger immune signalling [28-34].

We propose that functional specialization has been a critical event in NLR evolution that enhances NLR evolvability, i.e. their capacity to keep up with rapidly evolving pathogens without being constrained by signalling activity (Figure 1a). This view is supported by the emerging paradigm that many NLRs are interconnected not only in one-to-one relationship, but also through a complex network architecture [12]. Recently, a major clade of NLRs in Solanaceae plant species was shown to form an intricate immunoreceptor network. In this network, multiple helper NLRs, known as NLR-REQUIRED FOR CELLDEATH (NRC) are required by a large number of sensor NLRs that mediate resistance against diverse pathogens, such as viruses, bacteria, oomycetes, nematodes and insects [35]. Unlike typical paired NLRs, NRCs are not always clustered with sensor NLRs in plant genomes even though they are evolutionarily related [35]. The currentevolutionary model for the NRC network is that ithas evolved from a few genetically linked NLRs, as in the Caryophyllales, through massive expansion of sensors and a relatively limited expansion of helpers, possibly to maintain robustness of the network against rapidly evolving pathogens. As shown in Table 2, other NLR networks defined by the ubiquitous helper NLRs ADR1 and NRG1 have also been reported across several plantfamilies.

\section{NLRprotein complexes: assembling the immunoreceptor circuitry}

How do plant NLRs activate immunity after effector recognition? A common model is that plantNLRs, maintained in an inactive form through intramolecular associations, self-associate through their $\mathrm{N}$-terminal domains to trigger cell death and initiate immune signalling (Table 1) $[36,37]$. The TIR domain of a flax TIR-NLR L6, and Arabidopsis TIR-NLRs RPS4 and SUPPRESSOR OF npr1-1 CONSTITUTIVE 1 (SNC1) self-associate and is sufficient for HR cell death induction in planta $[38,39]$. Site-directed mutagenesis of the self-association interfaces disrupted signalling activity of the TIR domains and full-length TIR-NLRs [39]. The CC domain of CC-NLRs is also thought to mediate self-association to activate immunity. The CC domains of MLA10 and the wheat and rye orthologs, Sr33 and Sr50, can self-associate independently and trigger cell death in planta. Mutations in these CC domains abolished their selfassociation and induction of HR activity [40-42]. However, the greatest insight into CC domain function emerged from two recent ground-breaking studies on the structure and function of the ZAR1 CC-NLR $[6,14]$. Activation by the Xanthomonas campestris effector AvrAC triggers a series of molecular events through which an inactive monomer of ZAR1 forms a pentameric wheel-like complex termed a "ZAR1 resistosome" (Figure 1b) $[6,14]$. Remarkably, a stunning conformational switch — the death switch-releases the very terminal $\alpha 1$ helices of the CC 
domain, from being buried in the inactive ZAR1 to projecting out of the ZAR1 resistosome plane, to form a funnel-like structure. Structure-function studies revealed that the $\alpha 1$ funnel is required for cell death activity possibly by inserting into the plasma membrane and forming a toxic pore [6]. The structure of the activated ZAR1 resistosome takes us a leap forward in our understanding of CC-NLR activation with an emerging model of the CC domain directly exerting HR cell death through membrane perturbations without relying on downstream signalling pathway.

Not all CC domains appear to function as signalling domains. For instance, the CC domain of Arabidopsis CC-NLR RESISTANCE TO PSEUDOMONAS MACULICOLA 1 (RPM1) self-associates but does not trigger an immune response in planta [43]. Also, the $C C$ domain of potato $R x$ does not self-associate and fails to trigger HR $[44,45]$. We propose three possibilities to explain these inconsistencies. First, key residues or regions may be missing in the $\mathrm{CC}$ domains tested in self-association or functional assays, because different studies have used different domain boundaries and one amino acid difference of $\mathrm{CC}$ domain length can affect cell death inducing activity [40-42,46]. Second, other NLR domains (NB or LRR) could be involved in signalling along with the $\mathrm{CC}$ domain by supporting the overall protein complex integrity or by mediating subcellular localization. A recent finding demonstrated that a functional $p$-loop in the RPM1 NB domain is important for binding to the interactor RPM1-INTERACTING PROTEIN 4 (RIN4) and for proper subcellular localization [43]. Third, some CC domains may be nonfunctional relying on the $C C$ domains of their helper NLRs for function $[6,47]$. Thus, expression of $\mathrm{CC}$ domain truncations may be inadequately paired or may even lack their partners in heterologous expression systems. Therefore, going forward it is important to consider NLR activities in the context of intra- and inter-molecular connections between different NLR domains and appreciate that different $\mathrm{CC}$ domains may have distinct activities and functions.

Biochemical studies have revealed that several paired NLRs physically interact with each other for optimal function (Table 2). Arabidopsis RRS1/RPS4 and rice RGA5/RGA4 form homo-and hetero-complexes through their $\mathrm{N}$-terminal domains $[32,48]$. The interaction interface of the TIR domains of RRS 1 and RPS 4 is required for the immunoreceptor complex to respond to the pathogen effectors, AvrRps4 and PopP2 [48]. Recent large-scale interactome analyses revealed that N-terminal CC domains of Arabidopsis CC-NLR and RPW8-NLR (defined as "extended CC domains") are capable of forming highly redundant interaction networks suggestive of a high frequency of heteromeric interactions [46]. This biochemical interaction network is consistent with the emerging view that NLRs can form complex receptor network [12]. In Arabidopsis, the ACTIVATED DISEASE RESISTANCE 1 (ADR1) family (ADR1, ADR1-L1, ADR1-L2), members of the RPW8-NLR clade, are required by CC-NLRs (RPS2 and Rx2) and TIRNLRS [RESISTANCE TO PERONOSPORA PARASITICA 2 (RPP2), RPP4, SNC1, RRS1 and CHILLING SENSITIVE 1 (CHS1)/SUPPRESSORS OF chs1-2 3 (SOC3)] for immune response [49-52]. Another RPW8-NLR, known as N REQUIREMENT GENE 1 (NRG1), is necessary for full function of TIRNLRs [N, RECOGNITION OF XOPQ 1 (Roq1), WHITE RUST RESISTANCE 4A (WRR4A), WRR4B, RPP1,RPP2, RPP4, RRS1/RPS4, RRS1B/RPS4B, CHS1/SOC3and CHS3/CONSTITUTIVE SHADEAVOIDANCE 1 (CSA1)] and CC-NLRs (Rx2, RPS2 and RPM1) [50,52-55]. One hypothesis is that RPW8-NLR family members may have evolved as helper NLRs that co-operate with diverse sensor NLRs, including not only CC-NLRs but also TIR-NLRs. In the future, it would be interesting to match the genetic evidence for NLR networks with biochemical models and 
determine how NLR hetero-complexes are configured especially in light of the ZAR1 resistosome structural model (Figure 1b).

\section{NLR(mis)regulation: modulatingimmunity and autoimmunity}

NLR networks are regulated at different levels in plants. Plant NLRs are known to undergo trade-offs between disease resistance and growth and abiotic stress [56,57]. Mis-regulated NLRs trigger physiological phenotypes in plants including growth suppression known as the auto-immune phenotype [58-60]. In their resting state, NLRs are thought to be kept under check through chaperone complexes. For example, SKP1-CULLIN1-F-box (SCF) and other chaperone complexes control NLR protein levels and conformation in the absence of pathogen activation $[61,62]$.

Plant NLRs can also be tightly regulated at the transcriptional level [63]. Expression levels of some NLR genes are up-regulated in response to flg22, a bacterial microbe-associated molecular pattern (MAMP), or pathogen-related treatment $[64,65]$, suggesting that basal expression level of NLRs is kept low but is amplified in the cells attacked by plant pathogens (Figure 2a). MicroRNA-mediated gene silencing has been implicated in dynamic regulation of NLR transcripts [66]. Post-transcriptional NLR regulation by microRNAs such as miR482 superfamily is conserved in several plant species $[67,68]$. The miR482 level decreases in response to virus and bacterial pathogen infection, resulting in increased transcript levels of targeted NLRs [67]. Thus, the microRNA-mediated silencing machinery may contribute to suppress excessive accumulation of NLR transcripts and minimize fitness cost to the plant.

Interestingly, recent meta-analyses of $N L R$ gene expression profiles revealed that plant species have distinct tissue-specific expression patterns of NLR genes [69]. Transcriptome analyses across nine plant species revealed that monocot and Fabaceae plants preferentially express NLR genes in roots, but Brassicaceae plants, including Arabidopsis, show relatively high NLR expression in shoots [69]. These findings are consistent with the view that plants may have evolved tissue-specific NLR networks in a species-dependent manner, possibly to match the distinct pathogen attacks of different organs and tissues (Figure $2 \mathrm{~b}$ ). One model of tissue-specific NLR expression is epigenetic regulation of NLR promoters. A recent study focusing on the rice NLR Pigm cluster indicated that methylation levels of a transposable element in the Pigm promoter matched tissue-specific expression in pollen and leaves [70]. However, the regulatory mechanisms of $N L R$ gene expression and their functional relevance to disease resistance remain largely unknown.

Intra- and inter-molecular interactions form another layer of regulation of plant NLR activity. A widely cited model of intramolecular regulation is that the NB and LRR domains bind each other to suppress NLR activity in the absence of the pathogen [23,71,72]. Mutations in the NB or LRR domains of plant NLRs often confer auto-activity $[73,74]$. Consistently, the cryo-EM structure of ZAR1-RKS1 revealed that the LRR domain maintains ZAR1 in an inactive monomeric and ADP-bound state [14]. Intermolecular regulation between plant NLRs are known for NLR pairs. In some paired NLRs, such as RRS1/RPS4, RGA5/RGA4 and PigmS/PigmR, ectopic expression of helper NLRs results in auto-activity, which is suppressed by coexpressing the matching sensor NLRs $[32,70,75]$. In contrast, in the Pik-1/Pik-2 pair, helper NLR Pik-2 does not show autoimmunity in an ectopic expression system, and both NLRs are 
required to trigger an immune response in response to the matching effector $[33,76]$. Therefore, interconnections between sensor and helper NLRs are presumably regulated through different mechanisms depending on the paired genes. In line with this model, autoimmune responses are often observed at the F1 generation when wild Arabidopsis accessions are crossed-a phenomenon known as "hybrid necrosis" [59]. QTL mapping revealed that hybrid necrosis can be correlated with genetically unlinked NLR gene loci, for instance, between DANGEROUS MIX 1 (DM1) from Arabidopsis accession Uk-3 and DM2 from Uk-1 $[59,77,78]$. Interestingly, the auto-immune phenotype was triggered by hetero-complex of NLR proteins from the unlinked DM1 (DM1 ${ }^{\mathrm{Uk}-3}$ ) and DM2d (DM2 ${ }^{\mathrm{Uk}-1}$ ) alleles [79]. Thus, misregulated interconnections between genetically unlinked plant NLRs may rewire the immunoreceptor network resulting in auto-activation of the NLR complex (Figure 2c).

\section{Outlook: unravelling the genetic, biochemical and mechanistic links that define NLR immunoreceptor networks}

In this review, we highlight some of the recent findings on NLR biology by covering advances in NLR evolution, biochemical function sand genetic regulation. The emerging view is that NLRs form receptor networks that include not only singletons and pairs but also more complex connections that vary in their specificity and redundancy. It is likely that classic NLRs, such as MLA10, RPS5, ZAR1 and L6, function as singletons that do not require other NLRs to sense pathogens and activate immunity (Table 1, Box 1). In the ZAR1 resistosome, ZAR1 clearly functions as a singleton as it forms wheel-like pentamer in 1:1 relationship with its RLCK ligands RKS1/PBL2 ${ }^{\mathrm{UMP} P}$ and may trigger cell death directly through the funnel-shaped $\alpha 1$ helices of the CC domain [6]. However, other NLRs can be paired or networked and probably form various hetero-complexes to sense and respond to invading pathogens and pests (Table 2). Here, we propose an evolutionary model in which NLR singletons independently gave rise to functionally specialized NLRs that are connected in various ways (Figure 1a). Understanding the genetic, biochemical and mechanistic links between NLR network nodes should reveal a complex and dynamic architecture that would help to organize the hugely expanded NLRome of plants.

One pending question is the degree to which the metazoan inflammasome and apoptosome models apply to paired plant NLRs. In mammalian systems, NLR family CARD domaincontaining 4 (NLRC4)forms a wheel-like hetero-oligomercomplexwith the sensors NLR family apoptosis inhibitory proteins (NAIPs), to detect diverse ligands and recruit and activate Caspase-1 protease [80-82]. Whether or not sensor and helper NLRs form hetero-oligomer complexes similar to NLRC4/NAIPs is currently unknown (Figure 1b). A deeper mechanistic understanding of NLR networks is required and the recent resolution of the structure of the ZAR1 resistosome by Wang et al. [6] should pave the way to address this question.

Many other important questions remain unanswered. What is the molecular basis of the evolutionary transition from singletons to NLR pairs and networks, and how does this impact NLR protein functions and activities? What are the regulatory mechanisms of plant NLRs, and how do they differ between singleton and paired NLRs? How do sensor and helper NLRs function together, and do they form dynamic higher level resistosome-type complexes? What are the determinants of sensor/helper specificity in paired and network forming NLRs? 
Multidisciplinary approaches are required to dissect the evolution, assembly and regulation of the immune receptor circuitry of plants. But there is reason for optimism especially given the new conceptual framework provided by the elucidation of the structure and activation mechanism of the ZAR1 resistosome.

\section{Acknowledgements}

We are thankful to a number of colleagues for discussions and ideas. HA is funded by the Japan Society of the Promotion of Science (JSPS) and LD by a Marie Sklodowska-Curie Actions (MSCA) Fellowship. Our lab is funded primarily from the Gatsby Charitable Foundation, Biotechnology and Biological Sciences Research Council (BBSRC, UK), and European Research Council (ERC; NGRB and BLASTOFF projects). We thank Jian-Min Zhou and JiJie Chai for early access to the Wang et al. papers 


\section{Box 1. How to define singleton NLRs?}

Given our relatively limited mechanistic understanding of NLR activities, it is not straightforward to determine whether a given NLR protein functions as a singleton or whether it requires other NLRs. NLRs with integrated domains (NLR-IDs) are thought to be exclusively involved in pathogen sensing and those characterized so far have turned out to be associated with a paired NLR helper (aka executor) [27]. Classic NLRs, such as Rx, Bs2, Mi, Rpi-blb2, R1 and others, were first cloned as $R$ genes segregating for resistance against particular pathogens but turned out to require NLRs of the NRC helper class [35]. Typically, such NLRs cannot be transferred to taxonomically distinct plant families in the absence of their NLR mates [89]. This phenomenon quipped "restricted taxonomic functionality" by Brian Staskawicz and colleagues [90] may not apply to singleton NLRs, which tend to confer hypersensitivity when expressed alone in heterologous plants (Table 1). In contrast, the monocot CC-NLRs MLA10 and Sr50 can trigger HR cell death when co-expressed with matching effectors in Nicotiana benthamiana and their $\mathrm{CC}$ domains confer HR cell death when transiently expressed in this heterologous plant $[17,18,41,42]$. Although this test is not definitive and may depend on the degree of conservation of any endogenous helper NLRs (e.g. NRC and NRG1), it remains a first step to sort out NLRs into separate functional categories and dissect the NLRome network architecture of a given plant species.

\section{Figure Legends}

\section{Figure 1. Evolution of NLR networks.}

(a) NLR functions can be separated into pathogen detection termed "sensorfunction" and signalling or "helper (executor) function". In this model, singleton NLRs have both activities "sensor + helper". We propose that throughout evolution, some singleton NLRs have duplicated and functionally specialized into "sensors" and "helpers" forming connections that range from pairs to complex networks. (b) Possible resistosome configurations of sensor/helperNLR hetero-complexes. Onehypothesis is that the sensorNLRsengage into the resistosome as functional equivalents of RLCKs in the ZAR1 resistosome. Another possibility is that sensor NLRs are recruited as one of the wheel spokes in a hetero-oligomeric resistosome comprised of sensors and helper NLRs, as reported for the mammalian NAIP/NLRC4 inflammasome.

\section{Figure 2. Examples of NLR network regulation and mis-regulation.}

(a) Dynamic expression of NLR genes during pathogen infection results in a different network architecture throughout the host-pathogen interaction. (b) Tissue-specific NLR networks may have evolved to adapt to tissue-specific pathogen infections (e.g. nematodes in roots) and reduce the risk of autoimmunity. (c) Genetic crosses between distinct plant genotypes often cause incorrect connections between nodes in the NLR network, resulting in NLR misregulation and autoimmunity. 


\section{References}

1. Dodds PN, Rathjen JP: Plant immunity: towards an integrated view of plantpathogen interactions. Nat Rev Genet 2010, 11:539-548.

2. Win J, Chaparro-Garcia A, Belhaj K, Saunders DG, Yoshida K, Dong S, Schornack S, Zipfel C, RobatzekS, Hogenhout SA, etal.: Effector biology of plant-associated organisms: concepts and perspectives. Cold Spring Harb Symp Quant Biol 2012, 77:235-247.

3. Jones JDG, Vance RE, Dangl JL: Intracellular innate immune surveillance devices in plants and animals. Science 2016, 354.

4. Upson JL, Zess, EK, Bialas A, Wu CH, Kamoun S: The coming of age of EvoMPMI: evolutionary molecular plant-microbe interactions across multiple timescales. Curr Opin Plant Science 2018, 44:108-116.

5. Urbach JM, Ausubel FM: The NBS-LRR architectures of plant R-proteins and metazoan NLRs evolved in independent events. Proc Natl Acad Sci U S A 2017, 114:1063-1068.

6. Wang J, Hu M, Wang J, Qi J, Han Z, Wang G, QiY, Wang H-W, Zhou J-M, Chai J. Reconstitution and structure of a plant NLR resistosome conferring immunity. Science 2019, 364:eaav5870.

7. Bentham AR, BurdettH, AndersonPA, Williams SJ, KobeB:AnimalNLRs provide structural insights into plant NLR function. Ann Bot2017, 119:689-702.

8. Shao Z-Q, Xue J-Y, Wu P, Zhang Y-M, Wu Y, Hang Y-Y, Wang B, Chen J-Q: Large-scale analyses of angiosperm nucleotide-binding site-leucine-rich repeat genes reveal three anciently diverged classes with distinct evolutionary patterns. Plant Physiol 2016, 170:2095-2109.

9. Cesari S: Multiple strategies for pathogen perception by plant immune receptors. New Phytol 2018, 219:17-24.

10. Kourelis J, van der Hoorn RAL: Defended to the nines: 25 years of resistance gene cloning identifies nine mechanisms for R protein function. Plant Cell 2018, 30:285299.

11. Baggs E, Dagdas G, Krasileva KV: NLR diversity, helpers and integrated domains: making sense of the NLR IDentity. Curr Opin Plant Biol2017, 38:59-67.

12. Wu C-H, Derevnina L, Kamoun S: Receptor networks underpin plant immunity. Science 2018, 360:1300-1301.

13. Smakowska-Luzan E, Mott GA, Parys K, Stegmann M, Howton TC, Layeghifard M, Neuhold J, LehnerA, Kong J, Grünwald K, WeinbergerN, Satbhai SB, Mayer D, Busch W, Madalinski M, Stolt-Bergner P, Provart NJ, Mukhtar MS, Zipfel C, Desveaux D, Guttman DS, Belkhadir Y. An extracellular network of Arabidopsis leucine-rich repeat receptor kinases. Nature 2018, 18:342-346.

14. Wang J, Wang J, Hu M, W Shan, Qi J, Wang G, Han Z, QiY, Gao N, Wang H-W, Zhou J$\mathrm{M}$, Chai J. Ligand-triggered allosteric ADP release primes a plant NLR complex. Science 2019, 364:eaav5868.

15. Flor HH: Current status of the gene-for-gene concept. Annu Rev Phytopathol 1971, 9:275-296.

16. Maekawa T, Kracher B, Saur IML, Yoshikawa-Maekawa M, Kellner R, Pankin A, von KorffM, Schulze-lefertP:Subfamily-specific specialization of RGH1/MLAimmune receptors in wild barley. Mol Plant-Microbe Interact2018, 32:107-119. 
17. Saur IM, Bauer S, Kracher B, LuX, Franzeskakis L, Müller MC, Sabelleck B, Kümmel F, Panstruga R, Maekawa T, Schulze-LefertP. Multiple pairs of allelic MLA immune receptor-powdery mildew $\mathrm{AVR}_{\mathrm{A}}$ effectors argue for a direct recognition mechanism. Elife 2019, 19: e44471.

18. Chen J, Upadhyaya NM, OrtizD, Sperschneider J, LiF, Bouton C, BreenS, Dong C, Xu $B$, Zhang X, etal.: Loss of AvrSr50 by somatic exchange in stem rust leads to virulence for Sr50 resistance in wheat. Science 2017, 1610:1607-1610.

19. RavensdaleM, Bernoux M, Ve T, KobeB, ThrallPH, Ellis JG, Dodds PN: Intramolecular interaction influences binding of the flax $L 5$ and $L 6$ resistance proteins to their AvrL567 ligands. PLoS Pathog 2012, 8:e1003004.

20. Bernoux M, BurdettH, Williams SJ, ZhangX, Chen C, NewellK, Lawrence G, Kobe B, Ellis JG, Anderson P, etal.: Comparative analysis of the flax immune receptors $L 6$ and L7 suggests an equilibrium-based switch activation model. Plant Cell 2016, 28: 146-159.

21. Leonelli L, Pelton J, Schoeffler A, Dahlbeck D, Berger J, Wemmer DE, Staskawicz B. Structural elucidation and functional characterization of the Hyaloperonospora arabidopsidis effector protein ATR13. PLoS Pathog 2011, 7:e1002428.

22. Ade J, DeYoung BJ, Golstein C, Innes RW: Indirect activation of a plant nucleotide binding site-leucine-rich repeat protein by a bacterial protease. Proc Natl Acad Sci U S A 2007, 104:2531-2536.

23. QiD, DeYoungBJ, Innes RW: Structure-function analysis of the coiled-coiland leucine-rich repeat domains of the RPS5 disease resistance protein. Plant Physiol 2012, 158:1819-1832.

24. Baudin M, Hassan JA, Schreiber KJ, Lewis JD: Analysis of the ZAR1 immune complex reveals determinants for immunity and molecular. Plant Physiol2017, 174:20382053.

25. Gabriëls SHEJ, Vossen JH, Ekengren SK, van Ooijen G, Abd-El-Haliem AM, van den Berg GC, Rainey DY, Martin GB, Takken FL, de WitPJ, etal.: An NB-LRR protein required for $H R$ signalling mediated by both extra- and intracellular resistance proteins. Plant J 2007, 50:14-28.

26. Roberts M, Tang S, Stallmann A, Dangl JL, Bonardi V: Genetic requirements for signaling from an autoactive plant NB-LRR intracellular innate immune receptor. PLoS Genet2013, 9:e1003465.

27. Cesari S, Bernoux M, MoncuquetP, Kroj T, Dodds PN, Wise R, States U: A novel conserved mechanism for plant NLR protein pairs: the "integrated decoy" hypothesis. Front Plant Sci 2014, 5:1-10.

28. Narusaka M, Shirasu K, Noutoshi Y, Kubo Y, Shiraishi T, Iwabuchi M, Narusaka Y: RRS1 and RPS4 provide a dual Resistance-gene system against fungal and bacterial pathogens. Plant J 2009, 60:218-226.

29. Sarris PF, DuxburyZ, Huh SU, MaY, Segonzac C, Sklenar J, DerbyshireP, CevikV, Rallapalli G, Saucet SB, et al.: A plant immune receptor detects pathogen effectors that target WRKY transcription factors. Cell2015, 161:1089-1100.

30. Le Roux C, Huet G, Jauneau A, Camborde L, Trémousaygue D, Kraut A, Zhou B, Levaillant $\mathrm{M}$, Adachi $\mathrm{H}$, Yoshioka $\mathrm{H}$, etal.: A receptor pair with an integrated decoy converts pathogen disabling of transcription factors to immunity. Cell 2015, 161:1074-1088. 
31. Cesari S, Thilliez G, Ribot C, Chalvon V, Michel C, Jauneau A, Rivas S, Alaux L, Kanzaki $\mathrm{H}$, OkuyamaY, et al.: The rice resistance protein pair RGA4/RGA5 recognizes the Magnaporthe oryzae effectors AVR-Pia and AVR1-C039 by direct binding. Plant Cell 2013, 25:1463-1481.

32. Césari S, Kanzaki H, Fujiwara T, Bernoux M, Chalvon V, Kawano Y, ShimamotoK, DoddsP, Terauchi R, KrojT:TheNB-LRRproteinsRGA 4 andRGA5interact functionally and physically to confer disease resistance. EMBO J 2014, 33:19411959.

33. MaqboolA, Saitoh H, Franceschetti M, Stevenson CEM, UemuraA, KanzakiH, Kamoun S, Terauchi R, Banfield MJ: Structural basis of pathogen recognition by an integrated HMA domain in aplant NLR immune receptor. Elife 2015, 4:1-24.

34. DelaConcepcion JC, Franceschetti M, MaqboolA, SaitohH, TerauchiR, Kamoun S, Banfield MJ: Polymorphic residues in rice NLRs expand binding and response to effectors of the blast pathogen. Nat Plants 2018, 4:576-585.

35. Wu C-H, Abd-El-Haliem A, Bozkurt TO, BelhajK, Terauchi R, Vossen JH, Kamoun S: NLR network mediates immunity to diverse plant pathogens. Proc Natl Acad Sci U S A 2017, 114:8113-8118.

36. Ve T, Williams SJ, Kobe B: Structure and function of Toll/interleukin-1 receptor/resistance protein (TIR) domains. Apoptosis 2015, 1:250-261.

37. Bentham AR, ZdrzalekR, Dela Concepcion JC, Banfield MJ: Uncoiling CNLs: Structure/function approaches to understanding CC domain function in plant NLRs. Plant Cell Physiol 2018, 59:2398-2408.

38. BernouxM, VeT, WilliamsS, Warren C, ValkovE, ZhangX, EllisJG, Kobe B, Dodds PN: TIRdomain reveals interfaces for self-association, signaling, and autoregulation. Cell Host Microbe 2011, 9:200-211.

39. Zhang X, Bernoux M, Bentham AR, Newman TE, Ve T, Casey LW, Raaymakers TM, Hu $\mathrm{J}$, Croll TI, Schreiber KJ, et al.: Multiple functional self-association interfaces in plant TIR domains. Proc Natl Acad Sci U S A 2017, 114:E2046-E2052.

40. Maekawa T, ChengW, Spiridon LN, TöllerA, LukasikE, SaijoY, Liu P, Shen QH, Micluta MA, Somssich IE, et al.: Coiled-coil domain-dependent homodimerization of intracellular barley immune receptors defines a minimal functional module for triggering cell death. Cell Host Microbe 2011, 9:187-199.

41. Casey LW, Lavrencic P, Bentham AR, Cesari S, Ericsson DJ, Croll T, Turk D, Anderson PA, MarkAE, Dodds PN, etal.: The CC domain structure from the wheat stem rust resistance protein $\mathrm{Sr} 33$ challenges paradigms for dimerization in plant NLR proteins. Proc Natl Acad Sci U S A 2016, 113:12856-12861.

42. Cesari S, Moore J, Chen C, WebbD, PeriyannanS, Mago R, Bernoux M: Cytosolic activation of cell death and stem rust resistance by cereal MLA-family CC-NLR proteins. Proc Natl Acad SciU S A 2016, 113:10204-10209.

43. El Kasmi F, Chung E-H, Anderson RG, Li J, Wan L, Eitas TK, Gao Z, Dangl JL: Signaling from the plasma-membrane localized plant immune receptor RPM1 requires selfassociation of the full-length protein. ProcNatl Acad SciUS A2017, 114:E7385E7394.

44. Rairdan GJ, Collier SM, Sacco MA, Baldwin TT, Boettrich T, MoffettP: The coiled-coil and nucleotidebinding domains of the potato Rx disease resistance protein function in pathogen recognition and signaling. Plant Cell 2008, 20:739-751. 
45. HaoW, Collier SM, Moffett $P$, Chai J: Structural basis for the interaction between the potato virus $X$ resistance protein $(R x)$ and its cofactor Ran GTPase-activating protein 2 (RanGAP2). J Biol Chem 2013, 288:35868-35876.

46. Wróblewski T, Spiridon L, Martin EC, Petrescu AJ, Cavanaugh K, Truco MJ, Xu H, GozdowskiD, PawłowskiK, Michelmore RW, etal.: Genome-wide functional analyses of plant coiled-coil NLR-type pathogen receptors reveal essential roles of their N-terminal domain in oligomerization, networking, and immunity. PLoS Biol 2018, 16:e2005821.

47. AdachiH,Kamoun S, MaqboolA: Death switch: howresistosomes activate immunity. Zenodo2019, http://doi.org/10.5281/zenodo.2628967.

48. Williams SJ, Sohn KH, WanL, BernouxM, Sarris PF, Segonzac C, Ve T, MaY, Saucet $\mathrm{SB}$, Ericsson DJ, et al.: Structural basis for assembly and function of a heterodimeric plant immune receptor. Science 2014, 344:299-303.

49. Bonardi V, Tang S, Stallmann A, Roberts M, Cherkis K, Dangl JL: Expanded functions for a family of plant intracellular immune receptors beyond specific recognition of pathogen effectors. Proc NatI Acad SciU S A 2011, 108:16463-16468.

50. Collier SM, Hamel L-P, Moffett $P$ : Cell death mediated by the $N$-terminal domains of a unique and highly conserved class of NB-LRR protein. Mol Plant-Microbe Interact 2011, 24:918-931.

51. Dong OX, Tong M, Bonardi V, ElKasmi F, Woloshen V, WünschLK, DanglJL, LiX:TNLmediated immunity in Arabidopsis requires complex regulation of the redundant ADR1 gene family. New Phytol 2016, 210:960-973.

52. Wu Z, Li M, Dong OX, Xia S, Liang W, Bao Y, Wasteneys G, Li X: Differential regulation of TNL-mediated immune signaling by redundant helper CNLs. New Phytol2019, doi: 10.1111/nph.15665.

53. Peart JR, Mestre P, Lu R, Malcuit I, Baulcombe DC: NRG1, a CC-NB-LRR protein, together with N, a TIR-NB-LRR protein, mediates resistance against tobacco mosaic virus. Curr Biol 2005, 15:968-973.

54. Qi T, Seong K, Thomazella DPT, Kim JR, Pham J, Seo E, Cho M-J, Schultink A, Staskawicz BJ: NRG1 functions downstream of EDS1 to regulate TIR-NLR-mediated plant immunity in Nicotiana benthamiana. Proc Natl Acad Sci U S A 2018, 115:E10979-E10987.

55. Castel B, Ngou PM, Cevik V, Redkar A, Kim DS, Yang Y, Ding P, Jones JDG: Diverse NLR immune receptors activate defence via the RPW8-NLR NRG1. NewPhyto/2019, doi: 10.1111/nph.15659.

56. Karasov TL, Chae E, Herman JJ, Bergelson J: Mechanisms to mitigate the trade-off between growth and defense. Plant Cell 2017, 29: 666-680.

57. ArigaH, Katori T, Tsuchimatsu T, Hirase T, Tajima Y, Parker JE, Alcázar R, Koornneef $M$, Hoekenga O, Lipka AE, et al.: NLR locus-mediated trade-off between abiotic and biotic stress adaptation in Arabidopsis. Nat Plants 2017, 3:17072.

58. ShiranoY, KachrooP, Shah J, Klessig DF: Again-of-function mutation in an Arabidopsis Toll Interleukin1 receptor-nucleotide binding site-leucine-rich repeat type $\mathrm{R}$ gene triggers defense responses and results in enhanced disease resistance. Plant Cell 2002, 14:3149-3162.

59. Bomblies K, Lempe J, Epple P, Warthmann N, Lanz C, Dangl JL, Weigel D: Autoimmune response as a mechanism for a Dobzhansky-Muller-type incompatibility syndrome in plants. PLoS Biol2007, 5:1962-1972. 
60. Palma K, Thorgrimsen S, Malinovsky FG, Fiil BK, Nielsen HB, Brodersen P, Hofius D, Petersen M, Mundy J: Autoimmunity in Arabidopsis acd 11 is mediated by epigenetic regulation of an immune receptor. PLoSPathog2010, 6:e1001137.

61. Kadota Y, Shirasu K, Guerois R: NLR sensors meet at the SGT1-HSP90 crossroad. Trends Biochem Sci 2010, 35:199-207.

62. Cheng YT, Li Y, Huang S, Huang Y, Dong X, Zhang Y, Li X: Stability of plant immunereceptor resistance proteins is controlled by SKP1-CULLIN1-F-box (SCF)-mediated protein degradation. Proc Natl Acad SciU S A 2011, 108:14694-14699.

63. Lai Y, Eulgem T: Transcript-level expression control of plant NLR genes. Mol Plant Pathol 2018, 19:1267-1281.

64. Mohr TJ, Mammarella ND, HoffT, Woffenden BJ, Jelesko JG, McDowell JM: The Arabidopsis downy mildew resistance gene RPP 8 is induced by pathogens and salicylic acid and is regulated by w Box cis elements. MolPlant-Microbe Interact 2010, 23:1303-1315.

65. Yu A, Lepère G, Jay F, Wang J, Bapaume L, Wang Y, Abraham AL, Penterman J, Fischer RL, Voinnet $\mathrm{O}$, et al.: Dynamics and biological relevance of DNA demethylation in Arabidopsis antibacterial defense. Proc Natl Acad Sci U S A 2013, 110:2389-2394.

66. Park JH, Shin C: The role of plant small RNAs in NB-LRR regulation. BriefFunct Genomics 2015, 14:268-274.

67. Shivaprasad PV, Chen HM, Patel K, Bond DM, Santos BA, Baulcombe DC: A microRNA superfamily regulates nucleotide binding site-leucine-rich repeats and other mRNAs. Plant Cell 2012, 24:859-874.

68. Seo E, Kim T, Park JH, Yeom S-I, Kim S, Seo M-K, Shin C, Choi D: Genome-wide comparative analysis in Solanaceous species reveals evolution of microRNAs targeting defense genes in Capsicum spp. DNA Res 2018, 25:561-575.

69. Munch D, Gupta V, Bachmann A, Busch W, Kelly S, Mun T, Andersen SU: Organspecific NLR resistance gene expression varies with plant symbiotic status. bioRxiv 2017, doi: 10.1101/135764.

70. Deng Y,Zhai K, XieZ, Yang D, ZhuX, Liu J, Wang X, Qin P, Yang Y, Zhang G, etal.: Epigenetic regulation of antagonistic receptors confers rice blast resistance with yield balance. Science 2017, 965:962-965.

71. Slootweg EJ, Spiridon LN, Roosien J, Butterbach P, Pomp R, Westerhof L, Wilbers R, BakkerE,BakkerJ,Petrescu AJ, etal.: Structural determinants at theinterface of the ARC 2 and leucine-rich repeat domains control the activation of the plant immune receptors Rx1 and Gpa2. Plant Physiol2013, 162:1510-1528.

72. Schreiber KJ, Bentham A, Williams SJ, Kobe B, Staskawicz BJ: Multiple domain associations within the Arabidopsis immune receptor RPP1 regulate the activation of programmed cell death. PLoS Pathog 2016, 12:e1005769.

73. Bendahmane A, Farnham G, Moffett $P$, Baulcombe DC: Constitutive gain-of-function mutants in a nucleotide binding site-leucine rich repeat protein encoded at the $R \boldsymbol{x}$ locus of potato. Plant $\mathrm{J} 2002$, 32:195-204.

74. Tameling WI, Vossen JH, Albrecht M, Lengauer T, Berden JA, Haring MA, Cornelissen BJ, Takken FL: Mutations in the NB-ARC domain of I-2 that impair ATP hydrolysis cause autoactivation. Plant Physiol 2006, 140:1233-1245. 
75. Huh SU, Cevik V, Ding P, Duxbury Z, Ma Y, Tomlinson L, Sarris PF, Jones JDG: Proteinprotein interactions in the RPS4/RRS1 immune receptor complex. PLoSPathog 2017, 13:e1006376.

76. Zhai C, Lin F, Dong Z, HeX, Yuan B, Zeng X, Wang L, Pan Q: The isolation and characterization of $P i k$, a rice blast resistance gene which emerged after rice domestication. New Phytol 2011, 189:321-334.

77. ChaeE, Bomblies K, Kim ST, Karelina D, Zaidem M, Ossowski S, Martín-Pizarro C, Laitinen RA, Rowan BA, Tenenboim H, et al.: Species-wide genetic incompatibility analysis identifies immune genes as hot spots of deleterious epistasis. Cell 2014, 159:1341-1351.

78. AtanasovKE, Liu C, Erban A, Kopka J, Parker JE, AlcázarR: NLR mutations suppressing immune hybrid incompatibility and their effects on disease resistance. Plant Physiol 2018, 177:1152-1169.

79. Tran DTN, Chung EH, Habring-Müller A, Demar M, Schwab R, Dangl JL, Weigel D, Chae E: Activation of a plant NLR complex through heteromeric association with an autoimmune risk variant of another NLR. CurrBiol2017, 27:1148-1160.

80. Zhao Y, Yang J, Shi J, Gong YN, Lu Q, Xu H, Liu L, Shao F: The NLRC4 inflammasome receptors for bacterial flagellin and type III secretion apparatus. Nature 2011, 477:596-602.

81. Tenthorey JL, Kofoed EM, Daugherty MD, Malik HS, Vance RE: Molecular basis for specific recognition of bacterial ligands by NAIP/NLRC 4 inflammasomes. Mol Cell 2014, 54:17-29.

82. Tenthorey JL, Haloupek N, López-blanco JR, Grob P, Adamson E, Hartenian E, Lind NA, Bourgeois NM, Chacón P, NogalesE, etal.: Limit pathogen immune evasion. Science 2017, 358:888-893.

83. Lewis JD, Lee AH, Hassan JA, Wan J, Hurley B, Jhingree JR, Wang PW, Lo T, Youn JY, Guttman DS, et al.: The Arabidopsis ZED1 pseudokinase is required for ZAR1mediated immunity induced by the Pseudomonas syringae type III effector HopZ1a. Proc Natl Acad Sci U S A 2013, 110:18722-18727.

84. Wang G, RouxB, Feng F, Guy E, Li L, LiN, Zhang X, Lautier M, Jardinaud MF, Chabannes $M$, et al.: The decoy substrate of a pathogen effector and a pseudokinase specify pathogen-induced modified-self recognition and immunity in plants. Cell Host Microbe 2015, 18:285-295.

85. SetoD, Koulena N, LoT, Menna A, Guttman DS, DesveauxD: Expanded type III effector recognition by the ZAR1 NLR protein using ZED1-related kinases. Nat Plants 2017, 3:17027.

86. Narusaka M, Toyoda K, Shiraishi T, Iuchi S, Takano Y, Shirasu K, Narusaka Y: Leucine zipper motif in RRS1 is crucial for the regulation of Arabidopsis dual resistance protein complex RPS4/RRS1. Sci Rep 2016, 6:18702.

87. Zhang Y, Wang Y, Liu J, Ding Y, Wang S, Zhang X, Liu Y, Yang S: Temperaturedependent autoimmunity mediated by chs 1 requires its neighboring $T N L$ gene SOC3. New Phytol 2017, 213:1330-1345.

88. Xu F, Zhu C, Cevik V, Johnson K, Liu Y, Sohn K, Jones JD, Holub EB, Li X: Autoimmunity conferred by chs3-2D relies on CSA1, its adjacent TNL-encoding neighbour. Sci Rep 2015, 5:8792. 
89. Narusaka M, KuboY, Hatakeyama K, Imamura J, Ezura H, Nanasato Y, TabeiY, Takano Y, Shirasu K, Narusaka Y: Breaking restricted taxonomic functionality by dual resistance genes. Plant Signal Behav 2013, 8:e24244.

90. Tai TH, Dahlbeck D, Clark ET, Gajiwala P, Pasion R, Whalen MC, Stall RE, Staskawicz BJ: Expression of the Bs 2 pepper gene confers resistance to bacterial spot disease in tomato. Proc Natl Acad Sci U S A 1999, 96:14153-14158.

\section{References of special and outstanding interest}

*of special interest

Peart et al. 2005

This paperdescribes the first example of a genetic linkage between distinct NLRs. The authors used an unbiased virus-induced gene silencing screen of $N$. benthamiana cDNA library to show that the TIR-NLR N against Tobacco mosaic virus requires the RPW8-NLR protein, NRG1.

Shivaprasad et al. 2012

This paper experimentally demonstrates that a member of conserved miR482 superfamily targets NLR mRNA in N. benthamiana. Importantly, the authors show that miR482-mediated silencing machinery is suppressed in response to virus and bacterial infections, pointing to the role of miRNA in pathogen-inducible expression of NLR proteins.

Seo et al. 2018

Genome-wide comparative analysis of miRNA family members in Solanaceous species led to the identification of an miRNA subfamily targeting $N L R$ genes. The results suggest that miRn033 family exists in a common ancestor of Solanaceae plants, and species-specific miRNAs such as miR-n033a in pepper have evolved to regulate transcripts of expanded NLR genes in each plant species.

Munch et al. 2017

Transcriptome analysis across nine plant species reveal tissue-specific expression pattern of $N L R$ genes. Interestingly the authors show that NLR root/shoot expression ratios are different between monocot/Fabaceae plants and Brassicaceae plants.

Deng et al. 2017

This study describes epigenetic regulation of NLR pairs. Focusing on the rice NLR pair, PigmS/PigmR, the authors show thatPigmR constitutively expresses at low level, while PigmS shows tissue-specific expression pattern. PigmS directly suppresses PigmR function for rice blast resistance, but increases grain yield. Therefore, the authors propose that epigenetic regulation of an NLR pair balances disease resistance and fitness cost against yield. 
This paper describes the molecular basis of hybrid necrosis triggered by two genetically unlinked NLRs, DM1 from Arabidopsis accession Uk-3 and DM2 from Uk-1. These NLRs form a complex in planta and triggers autoimmunity in a heterologous plant system. The autoactive NLR complex does not require effector recognition for signalling activity. Therefore, some NLRs derived from different plant accessions lead to mis-regulated connection of NLR proteins, conferring NLRauto-activity.

${ }^{* *}$ of outstanding interest

Wu et al. 2017

A complex NLR network in Solanaceae and related plant species is described in this paper. Interestingly, helper NLRs, known as NRCs, are phylogenetically related to NRC-dependent sensor NLRs, forming a superclade. Moreover, comparative genomics suggest that the NRC network emerged over 100 Mya from an NLR pair.

Wróblewski et al. 2018

A large-scale interactome analysis using N-terminal extended CC (ECC) domains of Arabidopsis CC-NLRs and RPW8-NLRs suggests that CC-NLRs and RPW8-NLRs form biochemical interaction networks. This provides useful information on use of ECC domains for future biochemical studies.

Bonardi et al. 2011

ADR1 family members function to initiate immune signalling downstream of multiple sensor NLRs in Arabidopsis. The authors suggest the use of the term "helper NLR" to highlight a new category of NLRs specialized for signalling function.

Wu et al. 2019

This paper describes genetic requirements of NRG1 and ADR1 family genes for NLR-mediated signalling by using $n r g 1$ and adr1 triple mutants. The genetic evidence suggests that NRG1 and ADR1 function as helper NLRs for distinct TIR-NLR-triggered immune signalling in Arabidopsis.

Castel et al. 2019

In this paper the authors conducted Cas9-mediated mutagenesis on NRG1 in Arabidopsis and $N$. benthamiana. The set of NRG1 functional analyses including pathogen inoculation and HR cell death assays reveal that NRG1 is required for not only senor TIR-NLRs, but also TIR-NLR pairs and CC-NLRs in Arabidopsis. 
Wang etal.2019_Ligand-triggered allostericADP release primes a plantNLR complex.

This paperdescribes the first example of a full-length structure of a plant NLR. The authors used cryo-electron microscopy to solve Arabidopsis CC-NLR ZAR1 structure in complex with RKS1 or RKS1/uridylylated PBL2. Structural and biochemical analyses resulted in a mechanistic model of intramolecular regulation and ligand-mediated activation of ZAR1.

Wang et al. 2019_Reconstitution and structure of a plant NLR resistosome conferring immunity.

In this second paper on the ZAR1 resistosome, the authors revealed the cryo-electron microscopy structure of an activated Arabidopsis CC-NLRZAR1 complex. The complex forms a wheel-like pentamer with RKS1 and uridylylated PBL2. Structure-function analyses resulted in the model that ZAR1 undergoes a fold-switch that generates an $\mathrm{N}$-terminal funnel-shaped structure thatmay insert into the membraneand triggercell death through membrane pore toxic activity. 


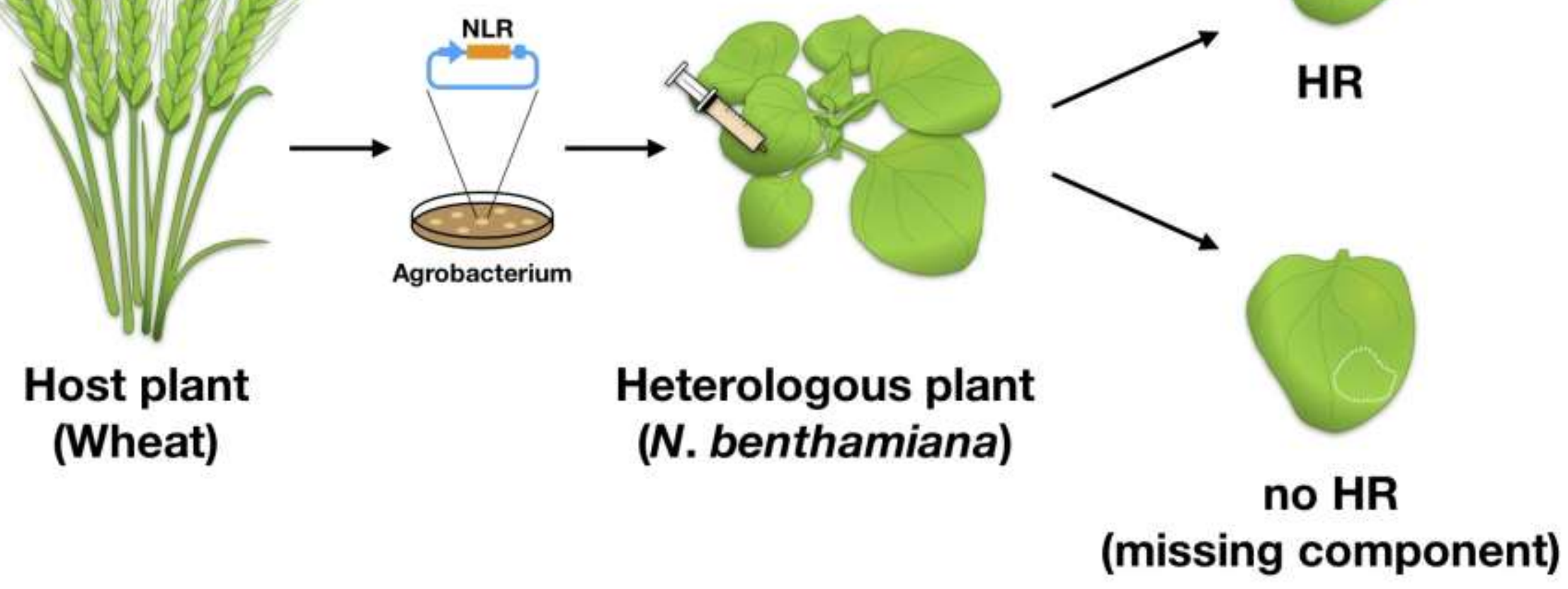


a

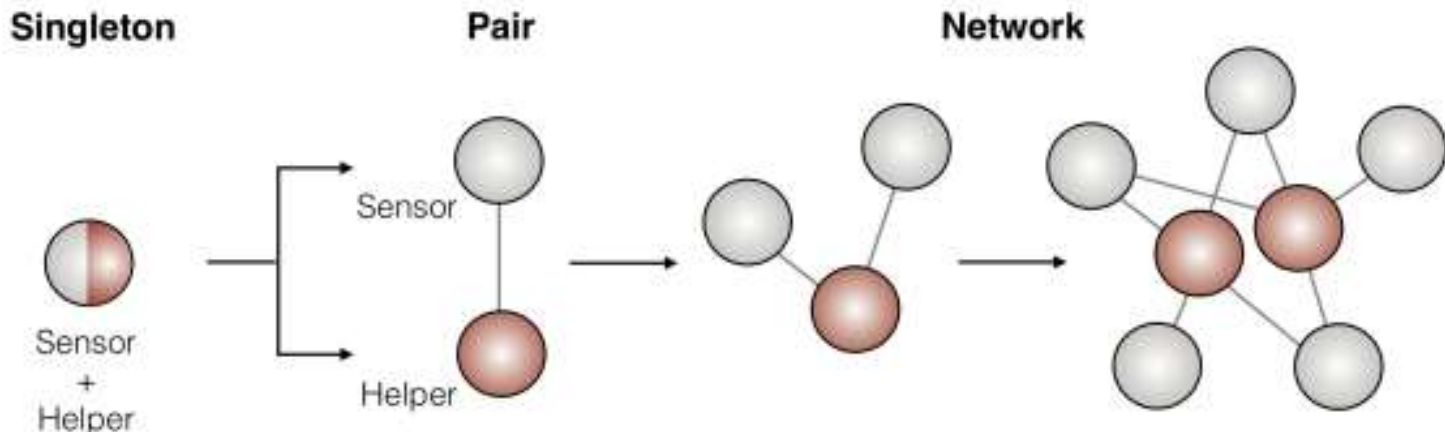

Specialization

Diversification

Time

b
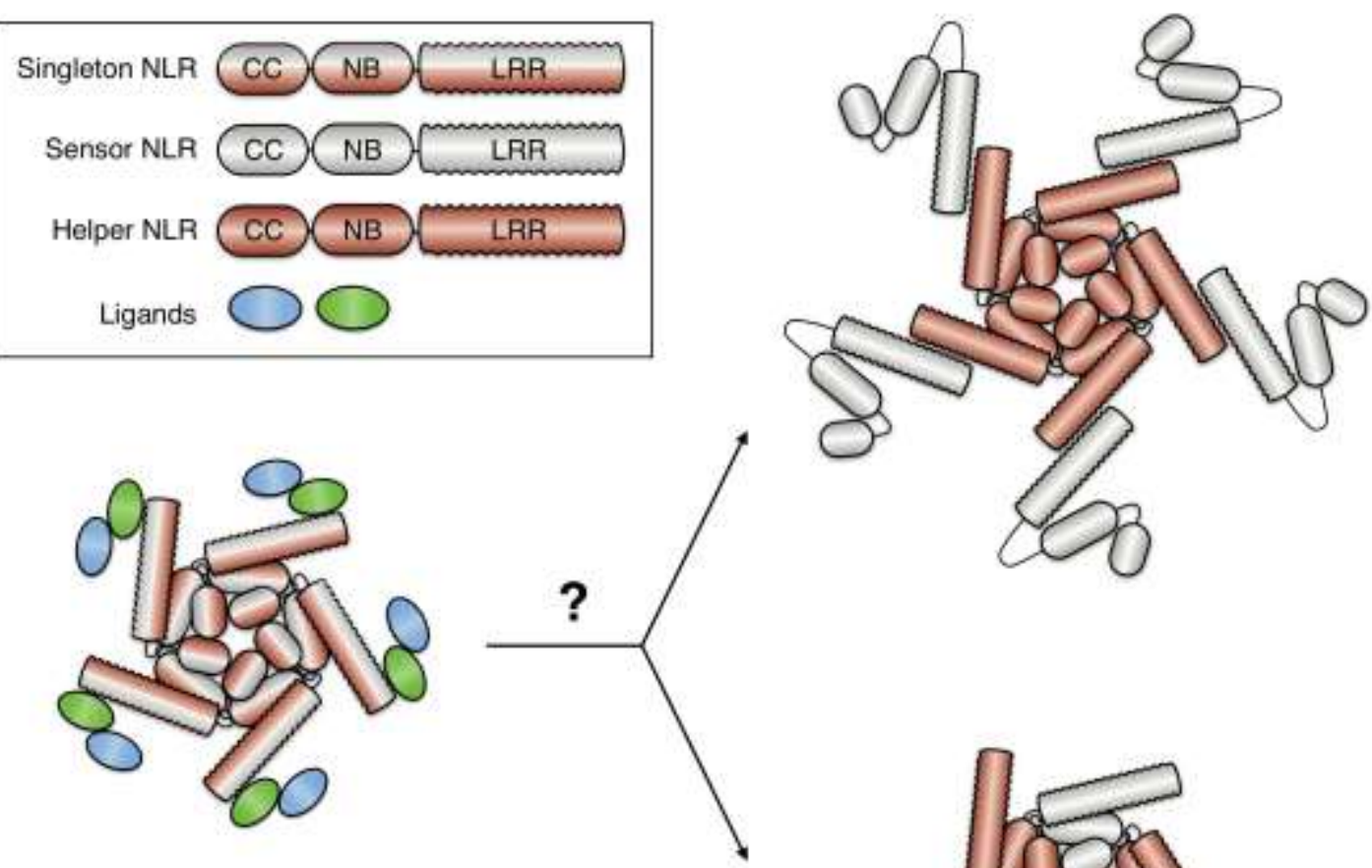

Singleton resistosome

Sensor + helper resistosome 
a

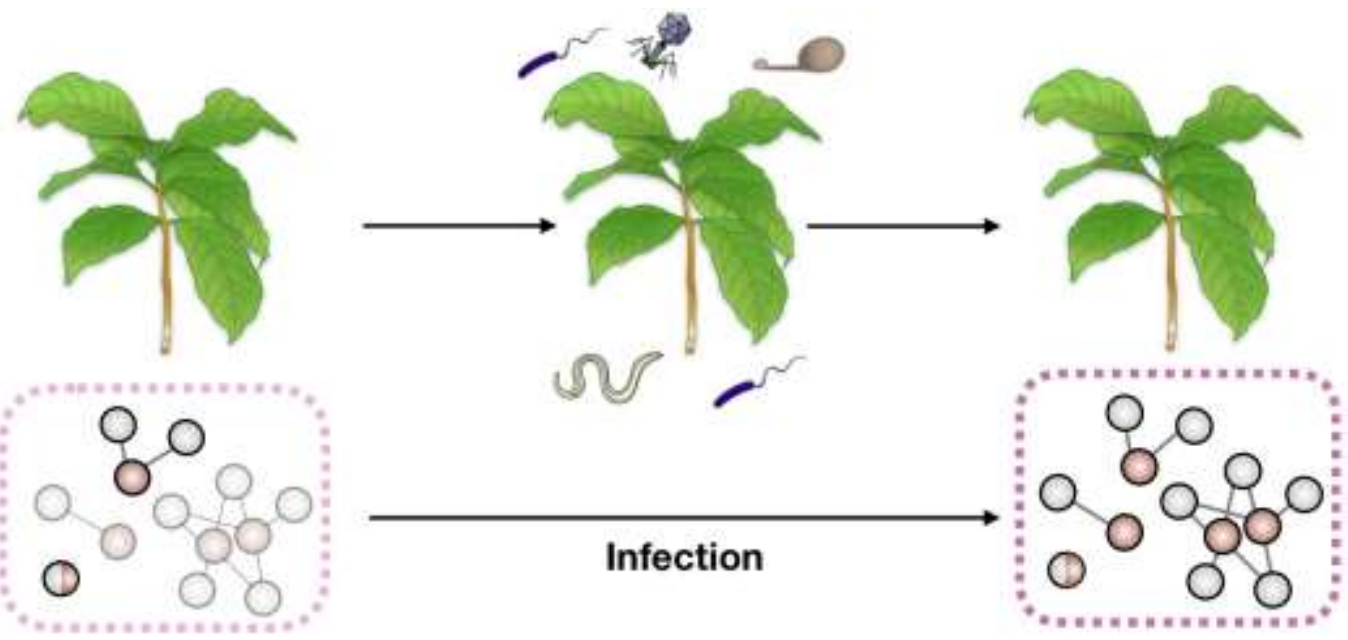

b

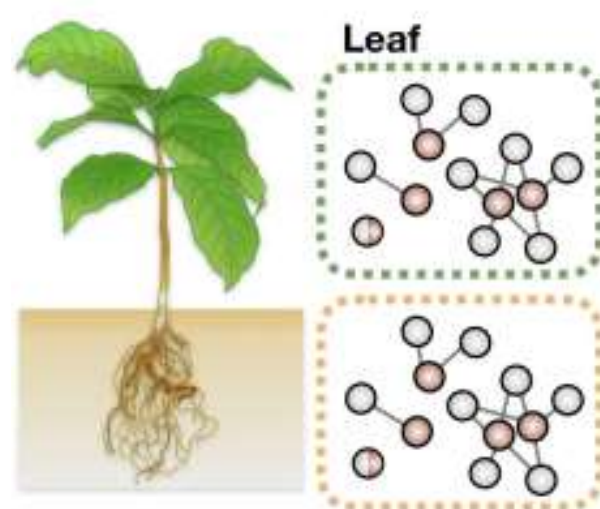

Root

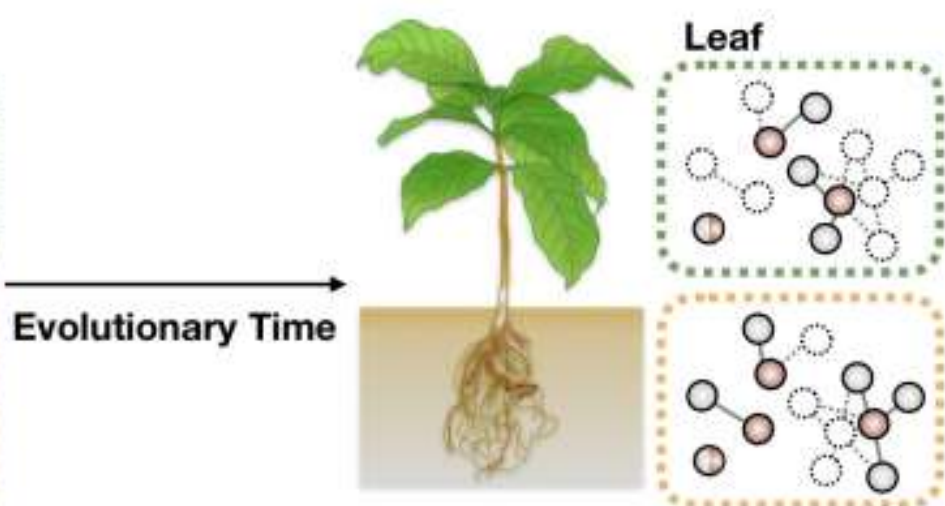

Root

C
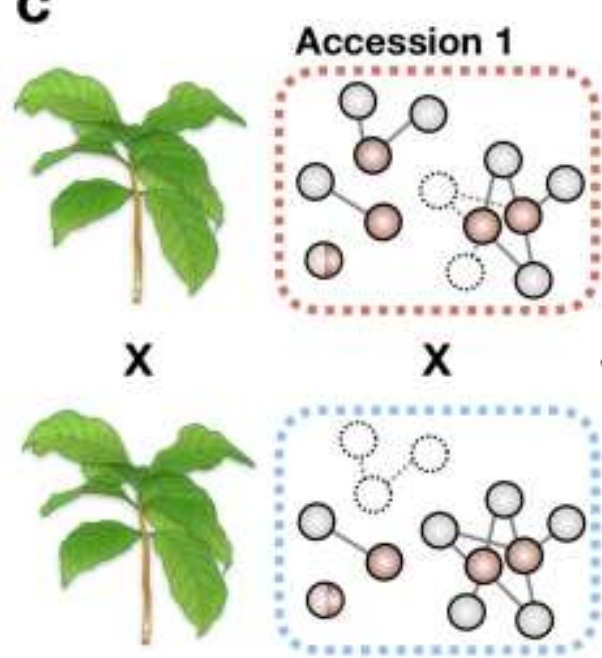

Accession 2

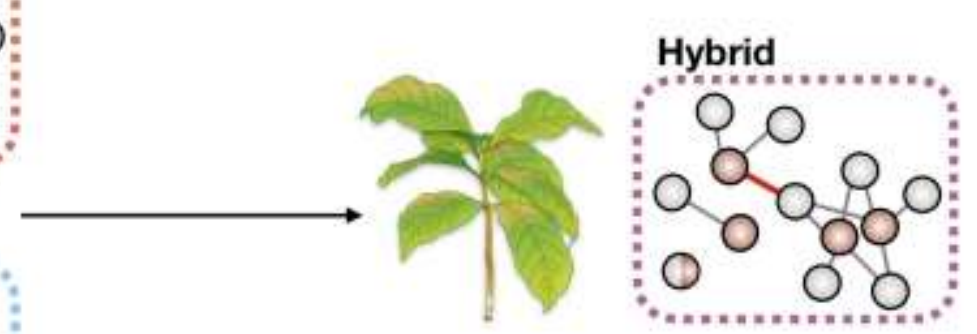

Autoimmunity by mis-connection

Singleton $O$ Sensor $O$ Helper 
Table 1. Examples of potential singleton NLRs

\begin{tabular}{|c|c|c|c|c|c|c|c|}
\hline Name & $\begin{array}{l}\text { NLR } \\
\text { type }\end{array}$ & $\begin{array}{l}\text { Host } \\
\text { organism }\end{array}$ & $\begin{array}{l}\text { HR in } \\
\text { heterologous } \\
\text { plant }\end{array}$ & Effector & Host target & $\begin{array}{l}\text { Self- } \\
\text { association }\end{array}$ & References \\
\hline MLA10 & $\mathrm{CNL}$ & Barley & Yes & AVRa10 & & Yes & 17,40 \\
\hline Sr50 & CNL & Rye & Yes & AvrSr50 & & Yes & $18,41,42$ \\
\hline RPS5 & $\mathrm{CNL}$ & Arabidopsis & Yes & AvrPphB & PBS1 & Yes & 22,23 \\
\hline ZAR1 & $\mathrm{CNL}$ & Arabidopsis & Yes & $\begin{array}{l}\text { HopZ1 } \\
\text { AvrAC } \\
\text { HopF2a }\end{array}$ & $\begin{array}{l}\text { ZED1 } \\
\text { RKS1/PBL2 } \\
\text { ZRK3 }\end{array}$ & Yes & $6,14,24,83-85$ \\
\hline L6 & TNL & Flax & Yes & AvrL567 & & Yes & $19,20,38$ \\
\hline
\end{tabular}

Table 2. Examples of NLR pairs and networks

\begin{tabular}{|c|c|c|c|c|c|c|c|}
\hline Sensor & NLR type & $\begin{array}{l}\text { Host } \\
\text { organism }\end{array}$ & Helper & NLR type & $\begin{array}{l}\text { Host } \\
\text { organism }\end{array}$ & $\begin{array}{l}\text { Biochemical } \\
\text { connection }\end{array}$ & References \\
\hline RGA5 & $\mathrm{CNL}$ & Rice & RGA4 & $\mathrm{CNL}$ & Rice & Yes & 32 \\
\hline Pik-1 & $\mathrm{CNL}$ & Rice & Pik-2 & $\mathrm{CNL}$ & Rice & NR & 33 \\
\hline PigmS & $\mathrm{CNL}$ & Rice & PigmR & CNL & Rice & Yes & 70 \\
\hline RRS1 & TNL & Arabidopsis & RPS4 & TNL & Arabidopsis & Yes & $39,48,86$ \\
\hline CHS1 & TNL & Arabidopsis & SOC3 & TNL & Arabidopsis & Yes & 87 \\
\hline CHS3 & TNL & Arabidopsis & CSA1 & TNL & Arabidopsis & NR & 88 \\
\hline $\mathrm{R} \times 2$ & $\mathrm{CNL}$ & $\begin{array}{l}\text { Solanaceae } \\
\text { spp. }\end{array}$ & ADR1 & RNL & $\begin{array}{l}\text { Solanaceae } \\
\text { spp. }\end{array}$ & NR & 50 \\
\hline $\begin{array}{l}\text { RPS2 } \\
\text { RPP2 } \\
\text { RPP4 } \\
\text { SNC1 } \\
\text { CHS1 (/SOC3) } \\
\text { RRS1 (/RPS4) }\end{array}$ & $\begin{array}{l}\text { CNL } \\
\text { TNL } \\
\text { TNL } \\
\text { TNL } \\
\text { TNL } \\
\text { TNL }\end{array}$ & Arabidopsis & $\begin{array}{l}\text { ADR1 } \\
\text { ADR1-L1 } \\
\text { ADR1-L2 }\end{array}$ & $\begin{array}{l}\text { RNL } \\
\text { RNL } \\
\text { RNL }\end{array}$ & Arabidopsis & NR & $49,51,52$ \\
\hline $\begin{array}{l}\text { Rx2 } \\
N \\
\text { Roq1 }\end{array}$ & $\begin{array}{l}\text { CNL } \\
\text { TNL } \\
\text { TNL }\end{array}$ & $\begin{array}{l}\text { Solanaceae } \\
\text { spp. }\end{array}$ & NRG1 & RNL & $\begin{array}{l}\text { Solanaceae } \\
\text { spp. }\end{array}$ & NR & $50,53,54$ \\
\hline $\begin{array}{l}\text { RPM1 } \\
\text { RPS2 } \\
\text { RPP1 } \\
\text { RPP2 } \\
\text { RPP4 } \\
\text { WRR4 } \\
\text { CHS1 (/SOC3) } \\
\text { CHS3 (/CSA1) } \\
\text { RRS1 (/RPS4) }\end{array}$ & $\begin{array}{l}\text { CNL } \\
\text { CNL } \\
\text { TNL } \\
\text { TNL } \\
\text { TNL } \\
\text { TNL } \\
\text { TNL } \\
\text { TNL } \\
\text { TNL }\end{array}$ & Arabidopsis & NRG1 & RNL & Arabidopsis & NR & $52,54,55$ \\
\hline $\begin{array}{l}\text { Bs2 } \\
\text { Prf } \\
\text { R8 } \\
\text { Rx } \\
\text { Sw5b }\end{array}$ & $\begin{array}{l}\mathrm{CNL} \\
\mathrm{CNL} \\
\mathrm{CNL} \\
\mathrm{CNL} \\
\mathrm{CNL}\end{array}$ & $\begin{array}{l}\text { Solanaceae } \\
\text { spp. }\end{array}$ & $\begin{array}{l}\text { NRC2 } \\
\text { NRC3 }\end{array}$ & $\begin{array}{l}\text { CNL } \\
\text { CNL }\end{array}$ & $\begin{array}{l}\text { Solanaceae } \\
\text { spp. }\end{array}$ & NR & 35 \\
\hline $\begin{array}{l}\text { Bs2 } \\
\text { CNL_11990 } \\
\text { Mi-1.2 } \\
\text { R1 } \\
\text { R8 } \\
\text { Rpi-blb2 } \\
\text { Rx } \\
\text { Sw5b }\end{array}$ & $\begin{array}{l}\mathrm{CNL} \\
\mathrm{CNL} \\
\mathrm{CNL} \\
\mathrm{CNL} \\
\mathrm{CNL} \\
\mathrm{CNL} \\
\mathrm{CNL} \\
\mathrm{CNL}\end{array}$ & $\begin{array}{l}\text { Solanaceae } \\
\text { spp. }\end{array}$ & NRC4 & $\mathrm{CNL}$ & $\begin{array}{l}\text { Solanaceae } \\
\text { spp. }\end{array}$ & NR & 35 \\
\hline
\end{tabular}


Conflict of interest

LD and SK have filed a patent application based on NLR networks. SK receives industry funding on NLR biology. 